\title{
HIGHER OBSTRUCTIONS TO DEFORMING COHOMOLOGY GROUPS OF LINE BUNDLES
}

\author{
MARK GREEN AND ROBERT LAZARSFELD
}

\section{INTRODUCTION}

Our purpose is to study the cohomological properties of topologically trivial holomorphic line bundles on a compact Kähler manifold. (See [GL1, GL2] for our prior work on this topic.)

Let $M$ be a compact connected Kähler manifold and, as usual, let $\operatorname{Pic}^{0}(M)$ denote the complex torus parametrizing isomorphism classes of topologically trivial holomorphic line bundles on $M$. We are interested in the analytic subvarieties $S^{i}(M), S_{m}^{i}(M) \subseteq \operatorname{Pic}^{0}(M)$ defined by

$$
\begin{aligned}
& S^{i}(M)=\left\{L \in \operatorname{Pic}^{0}(M) \mid H^{i}(M, L) \neq 0\right\}, \\
& S_{m}^{i}(M)=\left\{L \in \operatorname{Pic}^{0}(M) \mid h^{i}(M, L) \geq m\right\} .
\end{aligned}
$$

The study of these varieties was originally suggested by the work of Catanese [C1] and Beauville [B1], and we attempt to understand the questions that they asked in both [GL1] and this paper.

One of the main results of [GL1] was that if

$$
a_{M}: M \rightarrow \operatorname{Alb}(M)
$$

is the Albanese map of $M$, then $S^{i}(M)$ is a proper subvariety of $\operatorname{Pic}^{0}(M)$ if

$$
i<\operatorname{dim}\left(a_{M}(M)\right) \text {. }
$$

The technique was to study the first order deformation theory of the cohomology groups in question.

Here we consider the higher order deformation theory, and we find that all the higher obstructions to deforming a cohomology class vanish automatically along "straight lines" in $\operatorname{Pic}^{0}(M)$, where "straight line" means the image of a straight line in $H^{1}\left(M, \mathscr{O}_{M}\right)$ under the canonical exponential map

Received by the editors March 1, 1990.

1980 Mathematics Subject Classification (1985 Revision). Primary 14C30.

The first author's research was partially supported by NSF Grant DMS 88-02020.

The second author's research was partially supported by NSF Grant DMS 89-02551. 
$H^{1}\left(M, \mathscr{O}_{M}\right) \rightarrow \operatorname{Pic}^{0}(M)$. This leads to the following result:

Theorem 0.1. Let $M$ be a compact Kähler manifold, and $Z$ a positive-dimensional irreducible component of $S^{i}(M)$ or $S_{m}^{i}(M)$ for some $m$. Then

(1) $Z$ is a complex subtorus of $\operatorname{Pic}^{0}(M)$;

(2) There exists an analytic variety $N$ with $\operatorname{dim}(N) \leq i$ and a dominant analytic map $f: M \rightarrow N$ with connected fibers such that $Z \subseteq y+$ $f^{*}\left(\operatorname{Pic}^{0}(N)\right)$ for some $y \in \operatorname{Pic}^{0}(M)$;

(3) $N$ has maximal Albanese dimension, i.e. if $a_{N}$ is the Albanese map for any smooth model $\widehat{N}$ of $N$, then $\operatorname{dim}\left(a_{N}(\widehat{N})\right)=\operatorname{dim}(N)$.

When $i=1$, Beauville [B2] proved-as conjectured in [GL1]-that $S^{1}(M)$ consists of pullbacks of bundles from irrational pencils of genus $\geq 2$, together with a finite number of isolated points. " His method, involving a "twisted" version of Castelnuovo's Lemma about two 1-forms on a Kahler manifold that wedge to zero, is quite different from the techniques of this paper. However, when $i>1$, it is fairly easy (see Example 5.2) to construct examples where $M$ is the Cartesian product of a surface and a curve for which the line bundles in a positive-dimensional component of $S^{2}(M)$ do not pull back from a variety of dimension 1 or 2 ; such components consist of a constant line bundle tensored with a family that does pull back from a surface or a curve.

The proof of Theorem 0.1 builds on the deformation theory developed in our earlier work, but incorporates higher obstructions. The essential point is Theorem 3.2, in which we obtain a formula for computing locally the higher direct image sheaves of a family of topologically trivial line bundles. The result is that in the neighborhood of a given bundle $L \in \operatorname{Pic}^{0}(M)$, these direct images are computed by a complex $D_{L}^{\bullet}(\mathscr{L})$ constructed formally from the cohomology of $L$. As a matter of expository preference, we develop this obstruction theory in the context of the Dolbeault cohomology, but it is equally possible to work in a purely finite-dimensional setting.

In the situation of Theorem 0.1 , we have the inequalities

$$
\begin{aligned}
\operatorname{dim}\left(a_{M}(M)\right)-i & \leq \operatorname{dim}\left(a_{M}(M)\right)-\operatorname{dim}\left(a_{N}(N)\right) \\
& \leq \operatorname{dim}(\operatorname{Alb}(M))-\operatorname{dim}(\operatorname{Alb}(N)) \\
& \leq \operatorname{codim}(Z) .
\end{aligned}
$$

We therefore obtain our earlier result from [GL1]:

Corollary 0.2.

$$
\operatorname{codim}\left(S^{i}(M)\right) \geq \operatorname{dim}\left(a_{M}(M)\right)-i .
$$

Theorem 0.1 has some interesting applications. One is the problem of generalizing the Castelnuovo-DeFranchis lemma to the case when one has a compact

\footnotetext{
${ }^{1}$ Note added in proof. The results of [B1] have been supplemented and corrected in Beauville, "Annulation du $H^{1}$ pour les fibrés en droites plats," preprint.
} 
Kähler manifold $M$ and holomorphic forms $\omega \in H^{0}\left(\Omega_{M}^{1}\right)$ and $\beta \in H^{0}\left(\Omega_{M}^{i}\right)$ such that $\omega \wedge \beta=0$, but $\beta$ is not of the form $\omega \wedge \gamma$ for any $\gamma \in H^{0}\left(\Omega_{M}^{i-1}\right)$. We show in Theorem 5.3 that this forces $\omega$ to pull back from an analytic variety $N$ with $\operatorname{dim}\left(a_{N}(N)\right)=\operatorname{dim}(N) \leq i$ by an analytic map $f: M \rightarrow N$. There are easy examples on a product of three curves (Example 5.2) to show that $\beta$ does not necessarily pull back.

Another application is that one obtains restrictions on the fundamental group of compact Kähler manifolds. The main idea is that for any finitely presented group $G$, one can mimic the definition of $S^{1}(M)$ using group cohomology to define an object $S^{1}(G)$. These are subvarieties of the character group $\operatorname{Hom}\left(G, S^{1}\right)$ of $G$, and must turn out to be translates of subtori if $G=\pi_{1}(M)$ for a compact Kähler manifold $M$. These varieties are easily calculated given a presentation of the group, and rarely turn out to be subtori.

We want to mention that another interesting and closely related case in which higher obstructions are known to vanish automatically occurs in the work of Goldman-Millson [GM].

Both in originating the problem and at numerous steps along the way, Catanese and Beauville have inspired and influenced this work, both through their papers and private conversations on several continents. There has been a constant interweaving of our points of view, which in many ways, has resulted in a better paper.

\section{THE $\bar{\partial}$-OPERATOR FOR THE POINCARE BUNDLE}

We consider the Poincaré line bundle

$$
\mathscr{P} \stackrel{\pi}{\longrightarrow} M \times \operatorname{Pic}(M)
$$

(defined up to tensoring by line bundles on $\operatorname{Pic}(M)$ ), where $M$ is a compact Kähler manifold, and $\operatorname{Pic}(M)$ denotes some component of the Picard torus of $M$. Let $\phi_{1}, \phi_{2}, \ldots, \phi_{q}$ be harmonic $(0,1)$-forms on $M$ representing a basis for $H^{1}\left(M, \mathscr{O}_{M}\right)$. Choose a cover by polydiscs $U_{\alpha}$ for $M$. Let $y \in \operatorname{Pic}(M)$ and denote the corresponding line bundle by $L_{y} \rightarrow M$. Let $g_{\alpha \beta}$ be the transition functions for $L_{y}$ with respect to the cover $U_{\alpha}$.

The exponential sheaf sequence gives a canonical map

$$
H^{1}\left(M, \mathscr{O}_{M}\right) \stackrel{\tau}{\longrightarrow} \operatorname{Pic}^{0}(M) .
$$

If $\phi$ is a $\bar{\partial}_{M}$-closed $(0,1)$-form representing an element of $H^{1}\left(M, \mathscr{O}_{M}\right)$, then we may write

$$
\phi=\bar{\partial}_{M}\left(\lambda_{\alpha}\right) \text { on } U_{\alpha},
$$

where $\lambda_{\alpha}$ is a $\mathscr{C}^{\infty}$ function on $U_{\alpha}$. Then using the proof of the Dolbeault isomorphism, the transition functions $g_{\alpha \beta}$ for the bundle associated to $\tau(\phi)$ may be taken to be

$$
g_{\alpha \beta}=\exp \left(2 \pi \sqrt{-1}\left(\lambda_{\alpha}-\lambda_{\beta}\right)\right) .
$$


Proposition 1.1. Consider the map

$$
\mathbf{C}^{q} \stackrel{\tau_{y}}{\longrightarrow} \operatorname{Pic}(M)
$$

defined by

$$
\left(t_{1}, t_{2}, \ldots, t_{q}\right) \mapsto y+\tau\left(\sum_{i=1}^{q} t_{i} \phi_{i}\right)
$$

Let

$$
\phi_{i}=\bar{\partial}_{M}\left(\lambda_{i, \alpha}\right) \text { on } U_{\alpha} \text {, }
$$

where $\lambda_{i, \alpha}$ is a $\mathscr{C}^{\infty}$ function on $U_{\alpha}$. Then transition functions for $\left(\operatorname{id}_{M} \times \tau_{y}\right)^{*}(\mathscr{P})$ are

$$
\tilde{g}_{\alpha \beta}=\exp \left(2 \pi \sqrt{-1} \sum_{i=1}^{q} t_{i}\left(\lambda_{i, \alpha}-\lambda_{i, \beta}\right)\right) g_{\alpha \beta} .
$$

Further,

$$
\bar{\partial}_{L_{y+\tau\left(t_{1}, \ldots, t_{q}\right)}}=\bar{\partial}_{L_{y}}+\sum_{j} t_{j}\left(\wedge \phi_{j}\right) .
$$

Remark. Note that although $\mathscr{P}$ is only defined up to twists by line bundles on $\operatorname{Pic}(M)$, this ambiguity disappears when it is pulled back to $M \times \mathbf{C}^{q}$.

Proof. The formula for the transition functions follows from the formula given above for transition functions for $\tau(\phi)$.

For a general analytically varying family of analytic line bundles $L_{t}$, as treated in [GL2], we may write

$$
g_{\alpha \beta}(t)=\gamma_{\alpha}(t) g_{\alpha \beta}(0) \gamma_{\beta}(t)^{-1},
$$

where

$$
\gamma_{\alpha}(t): U_{\alpha} \rightarrow \mathbf{C}^{*}
$$

are $\mathscr{C}^{\infty}$ maps depending analytically on $t$. If

$$
\psi \in A^{0, i}\left(M, L_{t}\right),
$$

then we can represent $\psi$ by

$$
\psi_{\alpha} \in A^{0, i}\left(U_{\alpha}\right),
$$

satisfying

$$
\psi_{\alpha}=g_{\alpha \beta}(t) \psi_{\beta} .
$$

We may identify this with an element $\tilde{\psi} \in A^{0, i}\left(M, L_{0}\right)$ by taking

$$
\tilde{\psi}_{\alpha}=\gamma_{\alpha}(t)^{-1} \psi_{\alpha} \text {. }
$$

Now

$$
\bar{\partial}_{L_{t}} \tilde{\psi}_{\alpha}=\gamma_{\alpha}(t)^{-1} \bar{\partial}_{L_{0}}\left(\gamma_{\alpha}(t) \tilde{\psi}_{\alpha}\right) \text {. }
$$

Thus we have the fundamental formula:

$$
\bar{\partial}_{L_{t}}=\bar{\partial}_{L_{0}}+\gamma_{\alpha}(t)^{-1} \bar{\partial}_{L_{0}} \gamma_{\alpha}(t)
$$


In the case of the Poincare bundle above, we may apply this formula. Since in this case

$$
\gamma_{\alpha}(t)=\exp \left(\sum_{j} t_{j} \lambda_{j, \alpha}\right),
$$

we obtain the desired formula for the $\bar{\partial}$-operator, which completes the proof of the proposition.

\section{The Relative Dolbeault complex}

Let $M$ be a compact Kähler manifold; $Z$ a complex manifold; and $\mathscr{L} \rightarrow$ $M \times Z$ an analytic line bundle. Let $p_{1}: M \times Z \rightarrow M$ and $p_{2}: M \times Z \rightarrow Z$ be the canonical projections. Decompose the $\bar{\partial}$-operator on $\mathscr{L}$-valued $(0, \mathrm{i})$-forms as

$$
\bar{\partial}=\bar{\partial}_{M}+\bar{\partial}_{Z},
$$

where $\bar{\partial}_{M}$ comes by differentiation with respect to the $M$ variables and $\bar{\partial}_{Z}$ comes by differentiation with respect to the $Z$ variables. Now we introduce the sheaves $\mathscr{A}_{M \times \mathrm{Z} / \mathrm{Z}}^{0, i}(\mathscr{L})$ as defined by

$$
\mathscr{A}_{M \times Z / Z}^{0, i}(U, \mathscr{L})=\left\{\psi \in \Gamma\left(U, \mathscr{C}_{M \times Z}^{\infty}\left(p_{1}^{*} T_{M}^{(0, i) *} \otimes \mathscr{L}\right)\right) \mid \bar{\partial}_{Z} \psi=0\right\} .
$$

Lemma 2.1.

$$
\mathscr{R}_{p_{2} *}^{q}\left(\mathscr{A}_{M \times Z / Z}^{0, i}(\mathscr{L})\right)=0
$$

for all $i$ and all $q>0$.

Proof. This follows by using a partition of unity on $M$.

Lemma 2.2. The sequence

$$
0 \rightarrow \mathscr{O}(\mathscr{L}) \rightarrow \mathscr{A}_{M \times Z / Z}^{0,0}(\mathscr{L}) \stackrel{\bar{\partial}_{M}}{\longrightarrow} \mathscr{A}_{M \times Z / Z}^{0,1}(\mathscr{L}) \stackrel{\bar{\partial}_{M}}{\longrightarrow} \cdots
$$

is exact.

Proof. This is just the $\bar{\partial}$-Poincare lemma with analytic parameters. The same proof works.

Let

$$
A_{M \times Z / Z}^{0, i}(\mathscr{L})=p_{2 *} \mathscr{A}_{M \times Z / Z}^{0, i}(\mathscr{L})
$$

Note that

$$
A_{M \times Z / Z}^{0, i}(U, \mathscr{L})=\left\{\psi \in \Gamma\left(p_{2}^{-1}(U), \mathscr{C}_{M \times Z}^{\infty}\left(p_{2}^{*} T_{M}^{(0, i) *} \otimes \mathscr{L}\right)\right) \mid \bar{\partial}_{Z} \psi=0\right\} .
$$

This gives a complex of sheaves of $\mathscr{O}_{Z}$-modules $\left(A_{M \times Z / Z}^{0, \bullet}(\mathscr{L}), \bar{\partial}_{M}\right)$.

Proposition 2.3.

$$
\mathscr{R}_{p_{2 *}}^{i}(\mathscr{L}) \cong \mathscr{H}^{i}\left(A_{M \times Z / Z}^{0, \bullet}(\mathscr{L})\right) \text {. }
$$

Proof. The sheaves in the exact sequence of Lemma 2.2 are all sheaves of $p_{2}^{*} \mathscr{O}_{Z^{-}}$ modules, and $\bar{\partial}_{M}$ is a map of $p_{2}^{*} \mathscr{O}_{Z}$-modules. The proposition is a consequence 
of the spectral sequence relating the direct image sheaves of an exact sequence, taking note of the vanishing of the higher direct image sheaves of all but the left-most term.

We now take $Z$ to be the $n$-ball with coordinates $t_{1}, \ldots, t_{n}$, since our considerations are local. We make the assumption that we have a family of topologically trivial bundles, i.e. that

$$
c_{1}\left(\left.\mathscr{L}\right|_{M \times\{0\}}\right)=0 .
$$

The bundle $\mathscr{L} \rightarrow M \times Z$ gives an analytic map $f: Z \rightarrow \operatorname{Pic}^{0}(M)$ with $f(0)=$ $y$. Let $\phi_{1}, \ldots, \phi_{q}$ be a basis for the harmonic $(0,1)$-forms on $M$. We can write

$$
f(t)=\tau_{y}\left(\sum_{i=1}^{q} f_{i}(t) \phi_{i}\right),
$$

where the $f_{i}(t)$ are analytic functions vanishing at the origin. The computation of $\S 1$ gives that

$$
\bar{\partial}_{M}=\bar{\partial}_{L_{y}}+\bigwedge\left(\sum_{i} f_{i}(t) \phi_{i}\right)
$$

where the last term is to be interpreted as

$$
\psi \mapsto\left(\sum_{i} f_{i}(t) \phi_{i}\right) \wedge \psi
$$

We may summarize these remarks as

Proposition 2.4. In the situation just described, $A_{M \times Z / Z}^{0, \bullet}(\mathscr{L})$ is isomorphic to the complex

$$
\cdots A^{i-1}\left(L_{y}\right) \otimes \mathscr{O}_{Z} \longrightarrow A^{i}\left(L_{y}\right) \otimes \mathscr{O}_{Z} \longrightarrow A^{i+1}\left(L_{y}\right) \otimes \mathscr{O}_{Z} \cdots,
$$

with differential $\bar{\partial}_{L_{y}}+\Lambda\left(\sum_{i} f_{i}(t) \phi_{i}\right)$.

\section{THE DERIVATIVE COMPLEX OF SHEAVES AND DEFORMATIONS OF COHOMOLOGY GROUPS}

Let $M$ be a compact Kähler manifold and $\mathscr{L} \rightarrow M \times Z$ a family of topologically trivial analytic line bundles parametrized by the ball $Z$. Furthermore, let $t_{1}, \ldots, t_{n}$ be coordinates on $Z ; L_{t}=\left.\mathscr{L}\right|_{M \times\{t\}} ; L=L_{0}$ correspond to the point $y \in \operatorname{Pic}^{0}(M)$; and $\phi_{1}, \ldots, \phi_{q}$ be a basis for the harmonic $(0,1)$-forms on $M$. The map $t \mapsto L_{t}$ gives an analytic map $\tau: Z \rightarrow \operatorname{Pic}^{0}(M)$, which is given by

$$
t \mapsto \tau_{y}\left(\sum_{i} f_{i}(t) \phi_{i}\right),
$$

where the $f_{i}$ are analytic functions vanishing at the origin. 
The derivative complex of sheaves $D_{L}^{\bullet}(\mathscr{L})$ of the line bundle $\mathscr{L}$ at $L$ is the complex

$$
\cdots H^{i-1}(L) \otimes \mathscr{O}_{Z} \rightarrow H^{i}(L) \otimes \mathscr{O}_{Z} \rightarrow H^{i+1}(L) \otimes \mathscr{O}_{Z} \cdots,
$$

where the differential is a wedge product with

$$
\sum_{i} f_{i}(t) \phi_{i} \text {. }
$$

Lemma 3.1. Let $M$ be compact Kähler and $L \in \operatorname{Pic}^{0}(M)$. If $\phi \in A^{0,1}(M)$ and $\psi \in A^{0, i}(M, L)$ are harmonic, then $\phi \wedge \psi$ is harmonic, and

$$
[\phi] \wedge[\psi]=0 \text { in } H^{i+1}(M, L)
$$

if and only if

$$
\phi \wedge \psi=0 \text { in } A^{0, i+1}(M, L) .
$$

Proof. Since $L \in \operatorname{Pic}^{0}(M)$, it has a unitary flat connection. By Hodge theory for such bundles, when we conjugate we have

$$
\bar{\phi} \in H^{0}\left(M, \Omega_{M}^{1}\right) \text { and } \bar{\psi} \in H^{0}\left(M, \Omega_{M}^{i}\left(L^{*}\right)\right) .
$$

So $\bar{\phi} \wedge \bar{\psi}$ is holomorphic, and hence its conjugate $\phi \wedge \psi$ is harmonic. Now

$$
[\phi] \wedge[\psi]=0 \Leftrightarrow[\phi \wedge \psi]=0 \Leftrightarrow \phi \wedge \psi=0,
$$

which concludes the proof.

Theorem 3.2. Let $\mathscr{L} \rightarrow M \times Z$ be a family of topologically trivial line bundles on a compact Kähler manifold $M$ parametrized by the ball $Z$. Keeping the notations used above, we have

$$
\left(\mathscr{R}_{p_{2} *}^{i} \mathscr{L}\right)_{0} \cong\left(\mathscr{H}^{i}\left(D_{L}^{\bullet}(\mathscr{L})\right)\right)_{0},
$$

where the subscript 0 means stalk at zero.

Proof. Let $A^{\bullet}$ be the complex $A_{M \times Z / Z}^{0, \bullet}(\mathscr{L})$. We know that

$$
\mathscr{R}_{p_{2} *}^{i}(\mathscr{L}) \cong \mathscr{H}^{i}\left(A^{\bullet}\right) \text {. }
$$

Let $H_{L}^{\bullet}$ denote the complex

$$
\cdots H_{L}^{i-1} \otimes \mathscr{O}_{Z} \rightarrow H_{L}^{i} \otimes \mathscr{O}_{Z} \rightarrow H_{L}^{i+1} \otimes \mathscr{O}_{Z} \cdots,
$$

where $H_{L}^{i}$ represents the $L$-valued harmonic $(0, i)$-forms, and the differential is

$$
\wedge\left(\sum_{i} f_{i}(t) \phi_{i}\right) \text {. }
$$

This is a complex by Lemma 3.1 and a subcomplex of $A^{\bullet}$ by Proposition 2.4. There is a map of sheaves of $\mathscr{O}_{Z}$-modules

$$
\alpha_{i}: \mathscr{H}^{i}\left(H_{L}^{\bullet}\right) \rightarrow \mathscr{H}^{i}\left(A^{\bullet}\right)
$$


The kernel and cokernel of $\alpha_{i}$ are coherent sheaves. We want to show that $\alpha_{i}$ is an isomorphism on the stalks at 0 .

Let $\mathfrak{m}=\left(t_{1}, \ldots, t_{n}\right)$ denote the maximal ideal at 0 in $\left(\mathscr{O}_{Z}\right)_{0}$. This induces a filtration on $H_{L}^{\bullet}$ and $A^{\bullet}$. By the spectral sequences for these filtered complexes, we obtain filtrations on $\mathscr{H}^{i}\left(H_{L}^{\bullet}\right)$ and $\mathscr{H}^{i}\left(A^{\bullet}\right)$, which makes

$$
\alpha_{i}: \mathscr{H}^{i}\left(H_{L}^{\bullet}\right) \rightarrow \mathscr{H}^{i}\left(A^{\bullet}\right)
$$

into a filtered complex. The spectral sequence for this filtered complex gives a filtration on $\operatorname{ker}\left(\alpha_{i}\right)$ and $\operatorname{coker}\left(\alpha_{i}\right)$.

Let $\psi \in \operatorname{gr}^{p}\left(\operatorname{coker}\left(\alpha_{i}\right)\right)$ be a nonzero class. By the spectral sequence for the cohomology of the complex

$$
\alpha_{i}: \mathscr{H}^{i}\left(H_{L}^{\bullet}\right) \rightarrow \mathscr{H}^{i}\left(A^{\bullet}\right),
$$

this class is represented by a nonzero element of $\operatorname{gr}^{p}\left(\mathscr{H}^{i}\left(A^{\bullet}\right)\right)$. By the spectral sequence for the cohomology of the complex $A^{\bullet}$, this in turn is represented by a nonzero element of $\mathbf{g r}^{p}\left(A^{i}\right)$. Furthermore, any other element of $\mathfrak{m}^{p} A^{i}$ representing this class must be nonzero in $\mathbf{g r}^{p}\left(A^{i}\right)$. Thus we may represent $\psi$ by a form

$$
\sum_{I} \psi_{I} t^{I}
$$

where $\psi_{I}=0$ for $|I|<p$ and $\psi_{I} \neq 0$ for some $I$ with $|I|=p$. Let

$$
f_{i}(t)=\sum_{I} a_{i, I} t^{I}
$$

where we have $a_{i, 0}=0$. Since

$$
\bar{\partial} \psi_{I}+\sum_{i} \sum_{J+K=I} a_{i, J} \phi_{i} \wedge \psi_{K}=0,
$$

and the second term is zero if $|I|=p$, we conclude that both terms are zero in that case, and thus $\bar{\partial} \psi_{I}=0$ for $|I|=p$. For $|I|=p$, we may write

$$
\psi_{I}=\sigma_{I}+\bar{\partial} \mu_{I}
$$

where $\sigma_{I}$ is harmonic and $\mu_{I}$ is $\mathscr{C}^{\infty}$. We may now change $\psi$ by subtracting the differential of

$$
\sum_{|I|=p} \mu_{I} t^{I}
$$

so that $\psi_{I}=\sigma_{I}$ for $|I|=p$. The differential of

$$
\sum_{|I|=p} \sigma_{I} t^{I}
$$

is

$$
\sum_{I+i=J,|J|=p+1} \phi_{i} \wedge \sigma_{I} t^{J}=\sum_{|J|=p+1} \bar{\partial} \psi_{J} t^{J}
$$


Both sides of this equation are 0 since the left-hand side is harmonic and the right-hand side is $\bar{\partial}$-exact. Thus the differential of

$$
\sum_{|I|=p} \sigma_{I} t^{I}
$$

is 0 , and so we may change $\psi$ by

$$
\sum_{|I|=p} \sigma_{I} t^{I}
$$

so that now $\psi_{I}=0$ for all $I$ with $|I| \leq p$. This is a contradiction. We conclude that

$$
\operatorname{gr}^{p}\left(\operatorname{coker}\left(\alpha_{i}\right)\right)=0
$$

for all $p \geq 0$, and therefore $\operatorname{coker}\left(\alpha_{i}\right)_{0}=0$.

Now let $\psi \in \mathbf{g r}^{p}\left(\operatorname{ker}\left(\alpha_{i}\right)\right)$ be a nonzero class. By the same argument as for the cokernel, every element of $H_{L}^{i}$ representing $\psi$ must be nonzero in $\mathbf{g r}^{p}\left(H_{L}^{i}\right)$. Let $\psi$ be represented by an element,

$$
\sum_{I} \psi_{I} t^{I}
$$

Here $\psi_{I}$ is harmonic for all $I$ and $\psi_{I}=0$ for $|I|<p$, and some $\psi_{I_{0}} \neq 0$ for some $I_{0}$ with $\left|I_{0}\right|=p$. Further, there is a $\lambda \in A^{i-1}$ whose differential is $\psi$. We change $\lambda$ by the sum of the differential of an element of $A^{i-2}$ and something harmonic so as to make the smallest $|I|$ for which $\lambda_{I} \neq 0$ a number $k$ that is as large as possible. This does not change the class in $\operatorname{ker}\left(\alpha_{i}\right)$ represented by $\psi$. Note that $k$ exists and satisfies $k \leq p$, since otherwise $\psi$ would be zero in $\operatorname{gr}^{p}\left(H_{L}^{i}\right)$. If now $\lambda_{I}=0$ for $|I|<k$, and $\lambda_{I}$ is not harmonic for some $I$ with $|I|=k$, then $\bar{\partial} \lambda_{I}=\psi_{I}$ for $|I|=k$. Since one side of the equation is harmonic and the other is $\bar{\partial}$-exact, both are zero. We may now write $\lambda_{I}=\tau_{I}+\bar{\partial} \mu_{I}$ for all $I$ with $|I|=k$, where $\tau_{I}$ is harmonic and $\mu_{I}$ is smooth. We now change $\lambda$ by subtracting the harmonic form $\sum_{|I|=k} \tau_{I} t^{I}$ and subtracting the differential of $\sum_{|I|=k} \mu_{I} t^{I}$. We have now increased $k$, which is a contradiction.

Now let us take $Z$ to be one-dimensional and $\mathscr{L} \rightarrow M \times Z$ the bundle coming from the "straight line" $f: Z \rightarrow \operatorname{Pic}^{0}(M)$ given by

$$
f(t)=\tau_{y}(t \phi),
$$

where $\phi$ is a harmonic $(0,1)$-form. We have the derivative complex

$$
D_{\phi}^{\bullet}=D_{L}^{\bullet}(\mathscr{L}) \otimes \mathscr{O}_{Z} / \mathfrak{m}
$$

that is the complex of vector spaces

$$
\cdots \rightarrow H^{i-1}(L) \rightarrow H^{i}(L) \rightarrow H^{i+1}(L) \rightarrow \cdots,
$$

where the differential is $\wedge \phi$. This is the derivative complex considered in [GL1]. 
Corollary 3.3. Let $\mathscr{L} \rightarrow M \times Z$ be a "straight line" family of topologically trivial line bundles as above. Let $L_{t}=\left.\mathscr{L}\right|_{M \times\{t\}}$. Then on a neighborhood of 0 ,

$$
\mathscr{R}_{p_{2} *}^{i} \mathscr{L} \cong\left(K^{i} \otimes \mathscr{O}_{Z} / \mathfrak{m}\right) \oplus\left(H^{i}\left(D_{\phi}^{\bullet}\right) \otimes \mathscr{O}_{Z}\right),
$$

where $K^{i}$ is the kernel of the map

$$
H^{i}(L) \stackrel{\wedge \phi}{\longrightarrow} H^{i+1}(L) .
$$

In particular, there exists a punctured neighborhood $U$ of the origin in $Z$ so that

$$
H^{i}\left(L_{t}\right) \cong H^{i}\left(D_{\phi}^{\bullet}\right)
$$

for all $t \in U$.

Proof. Given any complex $V^{\bullet}$ of vector spaces with differentials

$$
A^{i}: V^{i} \rightarrow V^{i+1} \text {, }
$$

consider the complex

$$
\tilde{V}^{\bullet}=V^{\bullet} \otimes \mathbf{C}[t]
$$

with differentials

$$
\widetilde{A}^{i}=t A^{i}
$$

Then it is an elementary fact that

$$
H^{i}\left(\widetilde{V}^{\bullet}\right)=\left(\operatorname{ker}\left(A^{i}\right) \otimes \mathbf{C}[t] /(t)\right) \oplus\left(H^{i}\left(V^{\bullet}\right) \otimes \mathbf{C}[t]\right) .
$$

On a punctured neighborhood of zero, the sheaves $\mathscr{R}_{p_{2}{ }^{*}}^{i}(\mathscr{L})$ are all locally free, and therefore compute the pointwise cohomology groups $H^{i}\left(L_{t}\right)$.

\section{Proof of the MAIN THEOREM}

We are now ready to complete the proof of the main theorem. Let $M$ be a compact Kähler manifold and suppose that $m$ is a positive integer for which $\operatorname{dim} S_{m}^{i}(M)>0$ : fix some irreducible component $Z \subseteq S_{m}^{i}(M)$ of positive dimension. (Note that $S^{i}(M)=S_{1}^{i}(M)$, so that there is no loss in generality in treating this case.) There is a Zariski open subset $Z_{0}$ of $Z$ on which the dimension $h^{i}\left(M, L_{t}\right)$ is constant, and we may assume by changing $m$ if necessary that $h^{i}\left(M, L_{t}\right)=m$ for $t \in Z_{0}$. Fix any smooth point $y \in Z_{0}$, and given $\phi \in H^{1}\left(M, \mathscr{O}_{M}\right)$, denote by $D_{\phi}^{\circ}(y)$ the derivative complex that appears in Corollary 3.3, and by $l_{\phi}(y)$ the "straight line" through $y$ in the direction $\phi$. Then

$$
l_{\phi}(y):=\{y+\tau(t \phi) \mid t \in \mathbf{C}\} \subseteq \operatorname{Pic}^{0}(M) .
$$

If $T \subseteq Z$ is a smooth curve through $y$ with tangent vector $\phi$, then the function $h^{i}\left(M, L_{t}\right)$ is constant for $t \in T$ near $y$, and therefore, the differentials in $D_{\phi}^{\bullet}(y)$ vanish (c.f. [GL1, Theorem 1.6]). Thus,

$$
\phi \in T_{y}(Z) \Longleftrightarrow h^{i}\left(D_{\phi}^{\bullet}(y)\right) \geq m=h^{i}\left(M, L_{y}\right) .
$$


On the other hand, it follows from Corollary 3.3 that if $h^{i}\left(D_{\phi}^{\bullet}(y)\right) \geq m$, then $h^{i}\left(M, L_{t}\right) \geq m$ for every point $t \in l_{\phi}(y)$. Hence,

$$
\phi \in T_{y}(Z) \Rightarrow l_{\phi}(y) \subseteq Z .
$$

The fact that this is true for $y$ in a Zariski open subset of $Z$ implies that $Z$ is a translate of a subtorus of $\operatorname{Pic}^{0}(M)$.

Let $\hat{Z}$ be the dual torus to $Z$. Since $\operatorname{Alb}(M)$ is dual to $\operatorname{Pic}^{0}(M)$, the inclusion $Z \subseteq \operatorname{Pic}^{0}(M)$ yields by duality an analytic map $\pi: \operatorname{Alb}(M) \rightarrow \widehat{Z}$. Let

$$
u: M \rightarrow \hat{Z}
$$

be the composition of $\pi$ with the Albanese mapping $M \rightarrow \operatorname{Alb}(M)$, and $N$ be the image of the Stein factorization of $u$. Thus $N$ is a compact connected analytic variety admitting a finite mapping $N \rightarrow \widehat{Z}$, and one has an analytic map $f: M \rightarrow N$ with connected fibres. As $N$ maps finitely to a torus, it is clear that (any smooth model of) $N$ has maximal Albanese dimension. It remains to show that $N$ satisfies the other statements of Theorem 0.1 .

We argue first that $\operatorname{dim} N \leq i$. To this end, keeping the notation introduced at the beginning of the proof, fix harmonic $(0,1)$-forms

$$
\phi_{1}, \phi_{2}, \ldots, \phi_{k} \in A^{0,1}(M)
$$

on $M$ representing a basis for $T_{y}(Z)$, and let $\psi \in A^{0,1}\left(L_{y}\right)$ be a nonzero harmonic $L_{y}$-valued $(0, \mathrm{i})$-form on $M$. As we noted above, the fact that $\phi_{i} \in$ $T_{y}(Z)$ implies that the derivative complex $D_{\phi_{i}}^{\bullet}(y)$ has vanishing differentials, means that

$$
\phi_{j} \wedge \psi=0 \quad \forall j=1,2, \ldots, k .
$$

Equivalently, if

$$
\omega_{j}=\bar{\phi}_{j} \in H^{0}\left(M, \Omega_{M}^{1}\right) \text { and } \beta=\bar{\psi} \in H^{0}\left(M, \Omega_{M}^{i}\left(L^{*}\right)\right),
$$

then

$$
\omega_{j} \wedge \beta=0 \text { for } j=1,2, \ldots, k .
$$

Now consider a general point $x \in M$, and let

$$
r=\operatorname{dim}\left(\operatorname{span}\left(\omega_{1}(x), \omega_{2}(x), \ldots, \omega_{k}(x)\right)\right) .
$$

The map $u$ arises by integrating the one-forms $\omega_{i}$, and hence $\operatorname{rank}\left(d u_{x}\right)=r$, whence $r=\operatorname{dim} u(M)=\operatorname{dim}(N)$. On the other hand, if we trivialize $L_{y}$ near $x$, we may regard $\beta(x)$ as a nonzero element of $\wedge^{i} T_{x}^{*}(M)$ for which

$$
\omega_{j}(x) \wedge \beta(x)=0 \text { for } j=1,2, \ldots, k .
$$

It follows from the following elementary Lemma 4.1 that $r \leq i$, and thus $\operatorname{dim}(N) \leq i$, as claimed. 
Lemma 4.1 (c.f. [GL1]). Let $V$ be a finite-dimensional vector space, with $v_{1}, v_{2}, \ldots, v_{r}$ linearly independent elements of $V$. Let $0 \neq \beta \in \wedge^{i} V$ satisfy

Then $r \leq i$.

$$
v_{j} \wedge \beta=0 \text { for } j=1,2, \ldots, r \text {. }
$$

[Proof. Let $v_{1}, v_{2}, \ldots, v_{r}$ be part of a basis for $V$. One immediately sees that $\beta$ is of the form $v_{1} \wedge \cdots \wedge v_{r} \wedge \alpha$, from which the inequality follows.]

It remains to show that $Z \subseteq y_{0}+f^{*}\left(\operatorname{Pic}^{0}(N)\right)$ for some $y_{0} \in \operatorname{Pic}^{0}(M)$. In fact, it follows by double duality from the construction that the map $u: M \rightarrow \widehat{Z}$ induces on $\mathrm{Pic}^{0}$ an inclusion $v: Z=\operatorname{Pic}^{0}(\widehat{Z}) \rightarrow \operatorname{Pic}^{0}(M)$ that coincides with the original embedding $Z \subseteq \operatorname{Pic}^{0}(M)$ up to translation by some point $y_{0} \in$ $\operatorname{Pic}^{0}(M)$. By definition, however, $u$ factorizes as the composition $M \rightarrow N \rightarrow$ $\widehat{Z}$, and hence $v$ factors through the induced map $f^{*}: \operatorname{Pic}^{0}(N) \rightarrow \operatorname{Pic}^{0}(M)$. The assertion follows.

This completes the proof of the main theorem.

\section{EXAMPLES AND FURTHER APPLICATIONS}

Example 5.1. We start with an example to show that it is possible that a positivedimensional component of $S^{i}(M)$ is contained only in a translate of the pullback of $\mathrm{Pic}^{0}$ of a lower-dimensional variety. Let $X$ be an algebraic surface so that $S^{1}(X)$ consists of isolated points, and contains an element $L$ other than the trivial bundle (see [B1]). Note that $X$ cannot have any irrational pencils of genus $\geq 2$. Let $C$ be a curve of genus $\geq 2$, and put $M=C \times X$, with projections $p_{1}, p_{2}$. Thus $\operatorname{Pic}^{0}(M) \cong \operatorname{Pic}^{0}(C) \times \operatorname{Pic}^{0}(X)$. Then $S^{2}(M)$ contains $p_{1}^{*} S^{1}(C) \times p_{2}^{*} L$ as an irreducible component. By the remark above, it is impossible for this component to be the pullback by a map from $C \times X$ to a curve $C^{\prime}$ of some family of line bundles on $C^{\prime}$. If there were an analytic map $h: C \times X \rightarrow X^{\prime}$ to a surface $X^{\prime}$ so that the component of $S^{2}(M)$ just described pulls back from $\operatorname{Pic}^{0}\left(X^{\prime}\right)$, we would have a surjective map $\operatorname{Pic}^{0}\left(X^{\prime}\right) \rightarrow \operatorname{Pic}^{0}(C)$. Since $X$ has no irrational pencils of genus $\geq 2$, the map $X \rightarrow X^{\prime}$ must be dominant, so $\operatorname{Pic}^{0}\left(X^{\prime}\right) \rightarrow \operatorname{Pic}^{0}(X)$ must be injective. Up to isogeny, $\operatorname{Pic}^{0}(C)$ occurs as a factor of $\operatorname{Pic}^{0}(X)$. By varying $C$, we can be sure that this does not happen, as there are only countably many such factors. As a result, for manifolds of dimension $\geq 3$, Beauville's theorem [B2] does not hold in its full strength-there are positive-dimensional components of $S^{2}(M)$ that do not pull back from a curve or a surface. ${ }^{2}$ However, these components are a constant bundle tensored with a family of bundles that does pull back from a lower-dimensional variety. This explains the form taken by the main theorem.

A classical lemma of Castelnuovo and DeFranchis states that if $\omega$ and $\eta$ are linearly independent holomorphic one-forms on a compact Kahler manifold $M$

\footnotetext{
${ }^{2}$ See the footnote on page 2 .
} 
such that $\omega \wedge \eta=0$, then $\omega$ and $\eta$ pull back from a curve $C$ of genus $\geq 2$ under a surjective holomorphic map $f: M \rightarrow C$. As is clear from [GL1] and especially Beauville's work [B2], this lemma and its twisted analogue are closely related to the fact that any positive-dimensional component of $S^{1}(M)$ pulls back from a curve. Hence it is natural to ask whether there are analogues of Castelnuovo's lemma for forms of higher degree. The next example shows that the most naive possible generalization fails.

Example 5.2. Let $C_{1}, C_{2}, C_{3}$ be curves of genus $\geq 2$ and set $M=C_{1} \times$ $C_{2} \times C_{3}$, with $p_{1}, p_{2}, p_{3}$ the projections. Let $\lambda_{1}, \lambda_{2} \in H^{0}\left(\Omega_{C_{1}}^{1}\right)$ be linearly independent 1 -forms vanishing at exactly one common point, $\mu \in H^{0}\left(\Omega_{C_{2}}^{1}\right)$; and $\tau \in H^{0}\left(\Omega_{C_{3}}^{1}\right)$. Also let

$$
\begin{aligned}
& \beta=p_{1}^{*} \lambda_{1} \wedge p_{2}^{*} \mu+p_{1}^{*} \lambda_{2} \wedge p_{3}^{*} \tau \\
& \omega=p_{1}^{*} \lambda_{1}
\end{aligned}
$$

We notice that

$$
\omega \wedge \beta=0 .
$$

On the other hand, it is impossible to find a $\gamma \in H^{0}\left(\Omega_{M}^{1}\right)$ such that

$$
\beta=\omega \wedge \gamma,
$$

for then $\beta$ would vanish on $p_{1}^{-1}\left(\operatorname{div}\left(\lambda_{1}\right)\right)$, and this does not happen. Furthermore, $\beta$ does not pull back from an analytic map from $M$ to a surface, since then every component of the zero locus of $\beta$ would have dimension at least 1 , whereas $\beta$ vanishes at isolated points. Thus, the natural generalization of Castelnuovo's Lemma does not hold for $k$-forms with $k>1$. Notice, however, that $\omega$ does pull back from an analytic map from $M$ to a lower-dimensional variety. Indeed, this is essentially a general fact:

Theorem 5.3 (Generalized Castelnuovo Lemma). Let $M$ be a compact Kähler manifold of dimension $n$ and $L \in \operatorname{Pic}^{0}(M)$. Let $\omega \in H^{0}\left(\Omega_{M}^{1}\right)$. Consider the complex $A^{\bullet}$

$$
0 \rightarrow H^{0}(L) \stackrel{\wedge \omega}{\longrightarrow} H^{0}\left(L \otimes \Omega_{M}^{1}\right) \stackrel{\wedge \omega}{\longrightarrow} \cdots \stackrel{\wedge \omega}{\longrightarrow} H^{0}\left(X, L \otimes \Omega_{M}^{n}\right) \rightarrow 0 .
$$

Then

$$
H^{k}\left(A^{\bullet}\right)=0
$$

unless there exist a complex manifold $\tilde{N}$ of dimension $\leq k$ and a meromorphic map $f: M \rightarrow \widetilde{N}$ such that $\omega=f^{*} \tilde{\omega}$, where $\tilde{\omega} \in H^{0}\left(\boldsymbol{\Omega}_{\widetilde{N}}^{1}\right)$. Furthermore, $\widetilde{N}$ has the same dimension as the image of its Albanese map.

Remark. Referring to pulling back forms under a meromorphic map, we mean the following: By resolving the indeterminacies of $f$ one obtains a map $\tilde{f}: \widetilde{M} \rightarrow$ $\widetilde{N}$, with $\widetilde{M}$ smooth. The assertion is that $\omega$ pulls back to $\widetilde{M}$ from $\widetilde{N}$. Note that $H^{0}\left(M, \Omega_{M}^{1}\right)=H^{0}\left(\widetilde{M}, \Omega_{\widetilde{M}}^{1}\right)$. 
Proof. Let $\phi=\bar{\omega} \in H^{1}\left(\mathscr{O}_{M}\right)$ and $\psi=\bar{\beta} \in H^{k}\left(M, L^{*}\right)$ be an element representing a nonzero class in $H^{k}\left(\bar{A}^{\bullet}\right)$. If we deform the bundle $L$ on $M$ along the straight line in the direction $\phi$, the first obstruction to deforming the class $\psi$ vanishes. By Corollary 3.3, the entire straight line lies in $S^{i}(M)$, and so by the main theorem (and its proof) we obtain an analytic variety $N$ finite over a torus $\hat{Z}$, a map $f: M \rightarrow N$, and a one-form $\tilde{\omega}$ on $\hat{Z}$ that pulls back to $\omega$. If $\tilde{N}$ is a desingularization of $N$, then $\tilde{\omega}$ pulls back from $\operatorname{Alb}(\tilde{N})$ and hence from $\tilde{N}$ itself.

We remark that there is another direction in which one can generalize Castelnuovo's Lemma due to Ziv Ran [R]:

Ran's Theorem. Let $M$ be a compact Kähler manifold and $\omega_{1}, \omega_{2}, \ldots, \omega_{k+1}$ be linearly independent elements of $H^{0}\left(M, \Omega_{M}^{1}\right)$ such that

$$
\omega_{1} \wedge \omega_{2} \wedge \cdots \wedge \omega_{k+1}=0
$$

and so that no collection of $k$ linearly independent forms in the span of $\omega_{1}, \ldots$, $\omega_{k+1}$ wedges to zero. Then there exists a complex torus $T$ and an analytic map $f: M \rightarrow T$ such that $f(M)$ is a proper analytic subvariety of $T$ of dimension $\leq k$. Further, there exist $\lambda_{i} \in H^{0}\left(\Omega_{T}^{1}\right)$ such that $\omega_{i}=f^{*} \lambda_{i}$ for $i=1, \ldots, k+$ 1 .

There are some highly interesting results along these lines that follow from both Catanese [C2], who has applications to moduli of irregular varieties, and Peters, who has applications to infinitesimal Torelli for irregular varieties.

We now present some applications to the fundamental group of a compact Kähler manifold.

Definition. Let $G$ be a finitely presented group. For $\chi: G \rightarrow S^{1}$ a character, let $V_{\chi}$ be the associated $G$-module, so that $V_{\chi}$ is a one-dimensional complex vector space. The ith cohomological support varieties of $G$ are the sets

$$
\begin{aligned}
S^{i}(G) & =\left\{\chi \in \widehat{G} \mid H^{i}\left(G, V_{\chi}\right) \neq 0\right\}, \\
S_{m}^{i}(G) & =\left\{\chi \in \widehat{G} \mid h^{i}\left(G, V_{\chi}\right) \geq m\right\} .
\end{aligned}
$$

Lemma 5.4. Let $M$ be a compact Kähler manifold. Then if we let

$$
\tau: H^{1}\left(M, S^{1}\right) \rightarrow H^{1}\left(M, \mathscr{O}^{*}\right) \cong \operatorname{Pic}^{0}(M)
$$

denote the natural isomorphism, then

$$
S^{1}\left(\pi_{1}(M)\right)=\tau^{-1}\left(S^{1}(M) \cup-S^{1}(M)\right) .
$$

Proof. If $G=\pi_{1}(M)$, then associated to $\chi \in \widehat{G}$ we have the local system $\mathbf{L}_{\chi}$ on $M$. Recall then, that

$$
H^{1}\left(G, V_{\chi}\right) \cong H^{1}\left(M, \mathbf{L}_{\chi}\right)
$$


[In fact, let $\pi: \widetilde{M} \rightarrow M$ be the universal cover of $M$, and consider the double complex $K^{p, q}=C^{p}\left(G, A^{q}\left(\widetilde{M}, \pi^{*} \mathbf{L}_{\chi}\right)\right)$. The standard associated spectral sequences have

$$
\begin{aligned}
{ }^{\prime} E_{2}^{p, q} & =H^{p}\left(G, H^{q}\left(\widetilde{M}, \pi^{*} \mathbf{L}_{\chi}\right)\right) ; \\
{ }^{\prime} E_{2}^{p, q} & = \begin{cases}H^{q}\left(M, \mathbf{L}_{\chi}\right), & \text { if } p=0, \\
0, & \text { otherwise. }\end{cases}
\end{aligned}
$$

Since $H^{0}\left(G, H^{q}\left(\widetilde{M}, \pi^{*} \mathbf{L}_{\chi}\right)\right) \cong H^{q}(\widetilde{M}, \mathbf{C})^{G}$, we see that ${ }^{\prime} E_{2}^{p, 0} \cong H^{p}\left(G, V_{\chi}\right)$ for all $p$. Since also ${ }^{\prime} E_{2}^{0,1}=0$, the claim follows.]

On the other hand, we have the Hodge decomposition

$$
H^{1}\left(M, \mathbf{L}_{\chi}\right) \cong H^{1}\left(M, L_{\chi}\right) \oplus H^{0}\left(M, \Omega_{M}^{1} \otimes L_{\chi}\right),
$$

where $L \chi$ is the flat analytic vector bundle on $M$ associated to the local system $\mathbf{L}_{\chi}$; thus $L_{\chi}=\tau(\chi)$. Conjugation gives a complex anti-linear isomorphism $c: H^{0}\left(M, \Omega_{M}^{1} \otimes L_{\chi}\right) \cong H^{1}\left(M, L_{\chi}^{*}\right)$. The proposition now follows.

The following theorem was found by a different method by Gromov [Gr]. There is a related result of Siu [S] and Beauville.

Theorem 5.5. Let $M$ be a compact Kähler manifold, and $G$ a quotient of $\pi_{1}(M)$. Assume that $G$ has a presentation with $g$ generators and $r$ relations. If

$$
g \geq r+2
$$

then there exists an algebraic curve $C$ of genus at least $(g-r) / 2$ and a surjective analytic map $f: M \rightarrow C$. Moreover, if $G=\pi_{1}(M)$, then the image of the Albanese map $a: M \rightarrow \mathrm{Alb}(M)$ is a curve.

Proof. First we show that if $g \geq r+2$, then $S^{1}(G)=\widehat{G}$. To this end, suppose that $\chi: G \rightarrow S^{1}$ is a unitary character of $G$, and let $V_{\chi}$ be the corresponding one-dimensional representation. Recalling that

$$
Z^{1}\left(G, V_{\chi}\right)=\left\{f: G \rightarrow V_{\chi} \mid f(x y)=f(x)+\chi(x) f(y)\right\},
$$

we see that a one-cocycle for $G$ is determined by its values on the generators, and that each relation imposes at most one linear condition on these values. Hence

$$
\operatorname{dim} Z^{1}\left(G, V_{\chi}\right) \geq g-r .
$$

Since

$$
B^{1}\left(G, V_{\chi}\right)=\{(\chi-1)(\text { const })\}
$$

has dimension $\leq 1$, one has $\operatorname{dim} H^{1}\left(G, V_{\chi}\right) \geq g-r-1$ for every $\chi \in \widehat{G}$. Hence under the assumptions of the theorem, $S^{1}(G)=\widehat{G}$ as claimed.

Now suppose that $p: \pi_{1}(M) \rightarrow G$ is a surjective homomorphism. Then the induced map $p^{*}: H^{1}\left(G, V_{\chi}\right) \rightarrow H^{1}\left(\pi_{1}(M), V_{p^{*} \chi}\right)$ is automatically injective. It follows that

$$
\operatorname{dim}\left(S^{1}\left(\pi_{1}(M)\right)\right) \geq \operatorname{dim}(\widehat{G}) \geq(g-r) .
$$


This guarantees by the main theorem (or by Beauville's work) that there exists an analytic map $f: M \rightarrow C$ with $C$ a curve of genus at least $(g-r) / 2$. If $G=\pi_{1}(M)$, then

$$
S^{1}(M)=\operatorname{Pic}^{0}(M),
$$

and we know that this implies that the Albanese image of $M$ is a curve.

Theorem 5.6. Let $M$ be a compact Kähler manifold with $G=\pi_{1}(M)$. Then $S^{1}(G)$ and $S_{m}^{1}(G)$ for any $m \geq 1$ are unions (translates) of subtori in $\widehat{G}$.

Proof. Since $S^{1}(M)$ is a union of complex subtori of $\operatorname{Pic}^{0}(M)$, and $-S^{1}(M)$ also is, the result follows, since $\widehat{G} \cong \operatorname{Pic}^{0}(M)$.

\section{FURTHER REMARKS AND OPEN PROBLEMS}

Although in this paper we have concentrated on developing a higher obstruction theory for families of topologically trivial line bundles, there is a nice higher obstruction theory on compact Kähler manifolds for families of analytic vector bundles. For a family $V_{t}$ of vector bundles on $M$ parametrized by the disc, there is a spectral sequence $E_{r}^{p, q}$ such that

$$
E_{1}^{p, q} \cong H^{p+q}\left(V_{0}\right) \text { for } p \geq 0,
$$

and such that for $t$ in some punctured neighborhood of 0 ,

$$
E_{\infty}^{p, q} \cong H^{p+q}\left(V_{t}\right) \text { for } p>>0 .
$$

For a family of line bundles $L_{t}$, if

$$
\bar{\partial}_{M}=\bar{\partial}_{L_{0}}+\sum_{j} \phi_{j} t^{j}
$$

and if $\psi \in A^{0, i}\left(M, L_{0}\right)$ is a $\bar{\partial}$-closed form, then

$$
d_{1} \psi=\phi_{1} \wedge \psi \text {. }
$$

If this represents zero in cohomology, then

$$
\phi_{1} \wedge \psi=-\bar{\partial} \lambda_{1} .
$$

Then

$$
d_{2} \psi=\phi_{2} \wedge \psi+\phi_{1} \wedge \lambda_{1}
$$

If this is $-\bar{\partial} \lambda_{2}$, then

$$
d_{3} \psi=\phi_{3} \wedge \psi+\phi_{2} \wedge \lambda_{1}+\phi_{1} \wedge \lambda_{2},
$$

and so on. In the case where we consider "straight lines" in $\operatorname{Pic}^{0}(M)$, we may take all the $\lambda_{j}$ to be zero. Of course, it is usually quite subtle to compute the differentials of this spectral sequence when the line bundles are not topologically trivial. On the other hand, much of what we have done in this paper extends to the case of unitary flat vector bundles. 
Returning to the case of topologically trivial line bundles, if we consider

$$
S_{m}^{i, j}(M)=\left\{L \in \operatorname{Pic}^{0}(M) \mid h^{i}\left(\Omega_{X}^{j} \otimes L\right) \geq m\right\},
$$

then the spectral sequence for computing the higher obstructions is (after conjugating) the spectral sequence that appears in the proof of (3.4) in our paper [GL1]. This spectral sequence degenerates at the $E_{2}$ term, and this has the following consequence:

Theorem 6.1. Let $M$ be a compact Kähler manifold. Then every irreducible component of $S_{m}^{i, j}(M)$ is a complex subtorus of $\operatorname{Pic}^{0}(M)$.

The varieties $S_{m}^{i, j}(M)$ are no longer birational invariants of $M$. On the other hand, one might hope for some geometric explanation of them.

Another interesting question concerns the properties of the sheaves $\mathscr{R}_{p_{2 *}}^{i}(\mathscr{L})$ computed by the derivative complex of sheaves $D_{L}^{\bullet}(\mathscr{L})$. We would make the following conjecture:

Problem 6.2. If $\operatorname{dim}\left(a_{M}(M)\right)=\operatorname{dim}(M)$, then for the universal family of topologically trivial line bundles $\mathscr{L} \rightarrow M \times \operatorname{Pic}^{0}(M)$, is it true that

$$
\mathscr{R}_{p_{2} *}^{i}(\mathscr{L})=0
$$

for $i<\operatorname{dim}(M)$ ?

It would be interesting to have more examples of the varieties $S_{m}^{i}(G)$. In particular, are there some candidates for fundamental groups of compact Kähler manifolds that can only be eliminated by this method?

\section{BIBLIOGRAPHY}

[B1] A. Beauville, in Ueno (ed.), Classification of algebraic and analytic manifolds, Birkhäuser, 1983, pp. 619-620.

[B2] _ Annulation du $H^{1}$ et systèmes paracanoniques sur les surfaces, J. Reine Angew. Math. 388 (1988), 149-157.

[C1] F. Catanese, Moduli of surfaces of general type, Proc. of the 1982 Conf. at Ravello, Lecture Notes in Math., vol. 997, Springer-Verlag, 1983, pp. 90-112.

[C2] _ Moduli and classification of irregular Kaehler manifolds (and algebraic varieties) with Albanese general type fibrations, preprint.

[GL1] M. Green and R. Lazarsfeld, Deformation theory, generic vanishing theorems, and some conjectures of Enriques, Catanese, and Beauville, Invent. Math. 90 (1987), 389-407.

[GL2] __ A deformation theory for cohomology of analytic vector bundles on Kähler manifolds, with applications, Mathematical Aspects of String Theory, World Scientific, 1987, pp. 416440.

[GM] W. Goldman and J. Millson, The deformation theory of representations of fundamental groups of compact Kähler manifolds, Publ. Math. IHES 67 (1988), 43-96.

[Gr] M. Gromov, Sur le groupe fondamental d'une variété Kählerienne, preprint.

[R] Z. Ran, On subvarieties of abelian varieties, Invent. Math. 62 (1981), 459-479.

[S] Y. T. Siu, Strong rigidity for Kaehler manifolds and the construction of bounded holomorphic functions, Discrete Groups and Analysis, Birkhäuser, 1981, pp. 44-78.

Department of Mathematics, University of California at los Angeles, Los Angeles, CALIFORNIA 90024 